\title{
Riesgos estratégicos. Un estudio de las medidas de tratamiento implementadas por las grandes empresas privadas de Antioquia, Colombia
}

\author{
Strategic Risks. A Study of the Treatment Measures Implemented by the Big Private Companies in Antioquia, \\ Colombia
}

Riscos estratégicos. Estudo das medidas de tratamento implementadas pelas grandes empresas privadas de Antioquia, Colômbia

Isabel Jaraba

Universidad Eafit, Colombia

ijarabao@eafit.edu.co

ORCID: http://orcid.org/0000-0003-3734-8783

DOI: https://doi.org/10.11144/Javeriana.cc19-47.reem Redalyc: http://www.redalyc.org/articulo.oa?

$\mathrm{id}=151556994009$

María Antonia Nuñez

Universidad Eafit, Colombia

ORCID: http://orcid.org/0000-0002-1246-5361

Fecha de recepción: 08 Septiembre 2017

Fecha de aprobación: 18 Abril 2018

\author{
Eduart Villanueva \\ Universidad Eafit, Colombia \\ ORCID: http://orcid.org/0000-0003-4545-4025
}

\section{Resumen:}

Los cambios que enfrentan las organizaciones pueden poner en riesgo su normal desarrollo y afectar el cumplimiento de objetivos y metas. Por eso, es de vital importancia que estas puedan administrar esos riesgos adecuadamente mediante un proceso de identificación, evaluación y tratamiento. El objetivo de esta investigación fue analizar cómo las medidas de tratamiento utilizadas por las grandes empresas privadas de Antioquia permiten desarrollar conocimiento que faculta a los directivos para tomar decisiones que aporten al crecimiento de las organizaciones. Para cumplir con este propósito se realizó un estudio de carácter cualitativo, en el cual se examinaron diferentes fuentes de información documental relacionadas con el tema, con el fin de obtener instrumentos y herramientas que permitieran la apropiación de conocimiento. Igualmente, se aplicaron entrevistas semiestructuradas a altos directivos de empresas que cuentan con una estructura de administración de riesgos estratégicos. Los resultados de esta investigación aportan conocimiento sobre las medidas de tratamiento de riesgos estratégicos.

Palabras clave: riesgos estratégicos, administración de riesgos estratégicos, medidas de tratamiento, grandes empresas privadas.

\section{Abstract:}

The changes faced by the organizations may put at risk their normal development and affect the accomplishment of objectives and goals. Therefore, it is quite important that the organizations be able to manage those risk properly, through an identification, evaluation and treatment process. This research aimed to examine how the treatment measures used by big private companies in Antioquia allow to develop some knowledge empowering the directors for the decision-making, thus contributing to the organization growth. To attain this aim, a qualitative study was carried out examining different sources with documentary information related to this topic. This was intended to obtain instruments and tools enabling to appropriate this knowledge. In addition, semi-structured interviews were applied to top directors working in companies having a structure for the strategic risk management. The results from this research provide insights on the treatment measures to deal with strategic risks.

Keywords: strategic risks, strategic risk management, treatment measures, big private companies.

\section{Resumo:}

As mudanças enfrentadas pelas organizações podem colocar em risco o desenvolvimento normal e afetar o cumprimento de objetivos e metas. Portanto, é de vital importância para elas administrar esses riscos adequadamente mediante processos de identificação, avaliação e tratamento. O objetivo desta pesquisa foi analisar como as medidas de tratamento utilizadas pelas grandes empresas privadas de Antioquia permitem desenvolver conhecimento que faculta os diretivos na tomada de decisões a contribuir ao acréscimo das organizações. Para cumprir esse intuito realizou-se um estudo de carácter qualitativo, no que foram examinadas diferentes fontes de informação documental relacionadas com o tema, a fim de obter instrumentos e ferramentas que permitissem a apropriação de conhecimento. Igualmente, aplicaram-se entrevistas semiestruturadas a diretivos seniores de empresas que possuem 
uma estrutura de administração de riscos estratégicos. Os resultados desta pesquisa fornecem conhecimento sobre as medidas de tratamento de risco estratégico.

Palavras-chave: riscos estratégicos, administração de riscos estratégicos, medidas de tratamento, grandes empresas privadas.

\section{Introducción}

En el actual contexto empresarial, la incertidumbre es una constante que amenaza continuamente la estabilidad de las organizaciones (Louisot y Ketcham, 2014). Hay varios factores que pueden afectar el planteamiento estratégico y el logro de objetivos organizacionales de tipo político, económico, social, tecnológico y ambiental (Andersen y Schroeder, 2010; Kaplan y Norton, 2008). Además, influyen las fuerzas propias de cada sector y otros elementos de origen interno asociados al direccionamiento estratégico. También, la definición del modelo de negocio, la innovación y la adopción de nuevas tecnologías pueden afectar ese planteamiento y esos logros. Estos factores revelan la importancia de la administración de riesgos estratégicos, teniendo en cuenta que ella permite el adecuado manejo de las amenazas, antes de que estas se transformen en un problema real (Calandro, 2015).

El riesgo estratégico no administrado puede hacer que la estrategia empresarial fracase y que haya pérdidas que pueden llevar a la empresa a la quiebra (McConnell, 2016). Por tal razón, es importante seguir un proceso ordenado de identificación, evaluación y tratamiento, para contrarrestar los riesgos latentes (Calandro, 2015).

Debido a lo anterior, la investigación se propuso analizar cómo algunas de las grandes empresas privadas de Antioquia administran sus riesgos estratégicos, para así ahondar en el conocimiento de las medidas de tratamiento de este tipo de riesgos y mejorar su gestión. Para esto se identificaron las medidas de tratamiento que las empresas estudiadas usan para administrar sus riesgos estratégicos y se reconoció su proceso de implementación. Por ello se realizaron entrevistas semiestructuradas a siete directivos de grandes empresas privadas de Antioquia que cuentan con una estructura en administración de riesgos estratégicos.

El desarrollo de este estudio permitió aportar a la teoría acerca del riesgo en su ámbito estratégico. Esta investigación busca proporcionar herramientas que permitan tomar decisiones estratégicas y generar valor. Se quiere también usar tal conocimiento en la academia para el fortalecimiento de programas de pregrado, posgrado e investigación.

Se presenta, en primer lugar, un marco teórico acerca de la administración de riesgos estratégicos. Luego se expone la metodología de investigación, se procede a la presentación de los resultados obtenidos y posteriormente se muestran las conclusiones del estudio y las discusiones en torno al tema trabajado.

\section{Marco teórico}

En el presente marco teórico se parte del concepto de riesgo para proseguir con los planteamientos ofrecidos por diferentes autores que presentan el riesgo estratégico desde el punto de vista de la formulación y ejecución de la estrategia. Posteriormente se expone el proceso de la administración de riesgos estratégicos con sus diferentes etapas y se finaliza con la presentación de las medidas de tratamiento, que constituyen el foco de esta investigación, existentes en la literatura.

\section{Riesgo estratégico}

El riesgo, entendido como las eventualidades cuyas probabilidades son conocidas (Knight, 1921), posee componentes pasados, presentes y futuros, y tiene la posibilidad de menoscabar el valor de una empresa o, por el contrario, de traducirse en la creación de oportunidades (Hardy y Maguire, 2016; COSO, 2016; Beck, 
2002); además, puede presentarse en el nivel operativo en el normal desarrollo de las actividades (AIRMIC, Alarm y IRM, 2010) y también en el nivel estratégico durante la formulación, ejecución y modificación de la estrategia (Mejía, 2013). En este último nivel, el riesgo se articula con el desempeño a largo plazo e involucra factores internos y externos; asimismo, debe ser administrado por la alta dirección en un proceso continuo que involucra el establecimiento, ejecución y administración de la estrategia (Jnr y Pa, 2016).

Así pues, en la formulación de la estrategia, el riesgo significa la posibilidad de no identificar adecuadamente una posición de la empresa en la industria que sea tanto realizable como sostenible. Tener en cuenta esa posibilidad genera un proceso que brinda una seguridad razonable frente a la consecución de objetivos (McConnell, 2016; COSO, 2016). Desde la ejecución estratégica, los riesgos están constituidos por aquellos factores de alto nivel que pueden afectar el logro de los objetivos, y que de no ser identificados plenamente pueden hacerla vulnerable (McConnell, 2016). Estos riesgos pueden provenir de una sola decisión estratégica o de la sumatoria de políticas organizacionales que conducen a la toma de pequeños riesgos de manera inadecuada y afectar la continuidad de la empresa (Bromiley, Rau y McShane, 2016).

Por otro lado, los riesgos pueden proceder de variables internas o externas a la organización. Entre las primeras se encuentra la estrategia, concebida como el conjunto de lineamientos definidos en los objetivos, propósitos y metas que una organización espera cumplir, a partir de las políticas y planes establecidos, que son propios de cada organización y determinan el tipo de empresa y la actividad a la que se enfoca o se enfocará (Andrews, 1984). En el presente artículo se toma como modelo el reloj estratégico de Bowman (Johnson, Scholes, Whittington, López y Mazagatos, 2006), para identificar las diferentes opciones estratégicas de las empresas analizadas en dos dimensiones: valor percibido y precio, y a través de una matriz compuesta por ocho posibles estrategias permite identificar la posición competitiva de la empresa. De acuerdo con el modelo, una empresa puede partir de una estrategia de bajo costo y baja diferenciación, y en la medida en que el cliente percibe el valor añadido, aumentar el precio, lo que en la matriz supone moverse hacia la derecha en cada posición, de la siguiente manera:

1. Estrategia sin filigranas: parte de un bajo precio y un bajo valor añadido percibido por el cliente, que generalmente se enfoca en un mercado sensible al precio.

2. Estrategia de bajo precio: busca un precio inferior al de los competidores, al tiempo que se ofrece el mismo valor que ellos.

3. Estrategia híbrida: busca simultáneamente ofrecer productos y servicios diferenciados de los ofrecidos por los competidores y un precio más bajo que el de la competencia.

4. Estrategia de diferenciación: se ofrecen productos o servicios únicos y diferenciados de los ofrecidos por los competidores, por los cuales los clientes estén dispuestos a pagar más, lo que a su vez significa establecer precios superiores a los del mercado.

5. Diferenciación segmentada: busca diferenciarse mediante el enfoque en un determinado nicho de mercado y lograr un alto valor percibido por el cliente, lo que justifica un precio superior al de la competencia.

6. Las estrategias destinadas al fracaso, ubicadas en las posiciones seis, siete y ocho son aquellas en las cuales la organización disminuye valor mientras mantiene el precio, aumenta el precio mientras mantiene el valor o disminuye valor mientras aumenta el precio.

Así mismo, se encuentra el modelo de negocio, que contempla los fundamentos necesarios con los que una empresa presenta su propuesta de valor (Osterwalder y Pigneur, 2010), la cultura, que corresponde a una parte del contexto interno de la organización y consiste en las actitudes, experiencias y valores de las personas de la empresa, con el propósito de respaldar su misión y objetivos estratégicos (Pojasek, 2017), así como variables relacionadas con las innovaciones en productos, la adopción de nuevas tecnologías (Andersen y Schroder, 2010), la misión, la visión y la estructura organizacional (Mejía, 2013). Las segundas variables de carácter externo proceden del entorno político, económico, social, tecnológico y ambiental (Kaplan y 
Norton, 2008), y de otras fuerzas que establecen condiciones en cada sector, como son la rivalidad entre competidores actuales, el poder de negociación con que cuentan los clientes y proveedores, la amenaza del surgimiento de productos sustitutos y la amenaza de nuevos competidores (Porter, 1991).

\section{Administración de riesgos estratégicos}

Una vez examinado el concepto de riesgo estratégico, puede comprenderse que su administración debe convertirse en una habilidad primordial de la alta dirección (Frigo y Anderson, 2012), en pro de obtener altos rendimientos (Slywotzky, 2004), pues su adecuado análisis conduce a la disminución de la incertidumbre, a la toma de decisiones con mayor conocimiento (Edwards y Taborda, 2016) y a aprovechar las oportunidades derivadas de estas incertidumbres de manera exitosa (Bravo y Sánchez, 2009). En este sentido, la adecuada administración de los riesgos estratégicos debe ser un proceso continuo y sistemático (Frigo y Anderson, 2011), que debe estar integrado en la planeación estratégica, en el establecimiento de objetivos y en la medición del desempeño (Louisot y Ketcham, 2014; Kaplan y Norton, 2008).

La administración de riesgos estratégicos es un proceso dentro del cual debe establecerse el apetito de riesgo para la toma de decisiones (Calandro, 2015; Frigo y Anderson, 2009; Byrne y Durant-Law, 2007). En este sentido, el apetito de riesgo se define como la declaración de la cantidad de riesgo que la empresa está dispuesta a asumir y las pérdidas que podría aceptar en la ejecución de su estrategia (Calandro, 2015). Posteriormente deben identificarse, evaluarse, dar tratamiento y monitorear los riesgos presentes en las diferentes etapas de la estrategia (Jnr y Pa, 2016; Calandro, 2015; Frigo y Anderson, 2011; Frigo y Anderson, 2012).

La presente investigación se concentra en el tratamiento de los riesgos estratégicos como una etapa que, al ser propia de un riesgo relacionado con la estrategia, debe ser continua, dinámica y evolutiva (Frigo y Anderson, 2011). A nivel general, las medidas de tratamiento se traducen en acciones que mejoran las oportunidades y disminuyen las amenazas potenciales para la organización (Toulabi y Toulabi, 2013).

\section{Tratamiento de los riesgos estratégicos}

En forma generalizada, el tratamiento de los riesgos ha sido dividido en dos tipos de medidas: las de control, que consisten en prevenir, proteger y evitar; y las de financiación, que incluyen aceptar, retener y transferir (COSO, 2016; Toulabi y Toulabi, 2013; Mejía, 2013; AS NZS 4360, 2004). Para el presente marco teórico se incorpora el perseguir como medida de tratamiento (ISO 31000, 2018; COSO, 2016; AS NZS 4360, 2004) de los riesgos estratégicos.

Con relación a las medidas de control, prevenir hace referencia al desarrollo de medidas para la disminución de la frecuencia o probabilidad de ocurrencia del riesgo (Toulabi y Toulabi, 2013), proteger es disminuir el impacto del riesgo (Mejía, 2013), evitar consiste en no realizar los eventos potencialmente peligrosos (ISO 31000, 2018; COSO, 2016; Toulabi y Toulabi, 2013). En cuanto a las medidas de financiación, aceptar tiene que ver con no tomar medidas cuando la probabilidad y el impacto previstos son bajos y, por tanto, pueden asumirse (COSO, 2016; Toulabi y Toulabi, 2013). Por otro lado, transferir consiste en compartir el efecto de la materialización del riesgo con un tercero (COSO,2016; Calandro, 2015; Toulabi y Toulabi, 2013; AS NZS 4360, 2004). Finalmente, retener es crear un fondo o una reserva con el fin de prepararse para las pérdidas de manera anticipada (ISO 31000, 2018; Mejía, 2006; AS NZS 4360, 2004).

Ahora bien, perseguir se refiere a la búsqueda de mayores oportunidades por medio de la aceptación de mayores riesgos, al realizar actividades que puedan crear beneficios, adoptar estrategias agresivas y desarrollar nuevos productos, entre otros (COSO, 2016; AS NZS 4360, 2004). Una tarea importante de la alta dirección está relacionada con la aprobación de iniciativas empresariales que toman riesgos y con la formulación de una política relacionada con dicha toma de riesgos que garantice la alineación con los objetivos estratégicos y con 
las estructuras financieras, en la que se especifique el grado de riesgo que una empresa está dispuesta a aceptar en la búsqueda de sus objetivos generales (Andersen, Garvey y Roggi, 2014).

A nivel estratégico, el riesgo es susceptible de cambiar en cualquier momento, por estar ligado a la estrategia, que se ajusta al entorno de manera constante (Song y Yang, 2015); por lo tanto, con el tiempo puede cambiar el perfil de riesgo de las empresas, sin que se pueda mitigar (Calandro, 2015). Por lo expuesto anteriormente puede entenderse que el riesgo estratégico es propio de cada empresa, debido a que la estrategia, la cultura y el modelo de negocio obedecen a cada organización en particular (Deloitte, 2016).

\section{Metodología de investigación}

La presente investigación estudia las medidas de tratamiento de los riesgos estratégicos existentes en las grandes empresas privadas de Antioquia, Colombia. Para cumplir este objetivo, se utilizó un enfoque cualitativo, debido a que permite responder preguntas sobre el por qué y el cómo (Yin, 2003); las empresas bajo estudio utilizan los tratamientos para estos riesgos; además, permite entender las experiencias y aprendizajes de los entrevistados (Rubin y Rubin, 1995) durante el proceso de reconocimiento e implementación de las medidas de tratamiento.

El alcance de la investigación es descriptivo, en el que se relacionan las características específicas sobre la forma como las grandes empresas privadas responden ante los riesgos estratégicos. El diseño de la investigación es no experimental y transversal, debido a que se requería estudiar el fenómeno en sus condiciones naturales, sin manipular las variables y en un solo momento del tiempo (Hernández, Fernández y Baptista, 2010).

El instrumento de recolección de información es la entrevista semiestructurada, que mediante un protocolo previamente diseñado a partir de las categorías de análisis derivadas de la literatura y la inclusión de preguntas adicionales surgidas de la conversación (Erlandson, Harris, Skipper y Allen, 1993), permiten obtener mayor información (Vargas-Jiménez, 2012), dirigir la entrevista hacia los aspectos relevantes del estudio y poder profundizar en ellos (Rubin y Rubin, 1995). Dicho instrumento fue validado con una prueba piloto en una gran empresa privada, que permitió observar si las preguntas eran claras y tenían coherencia con el propósito del estudio; la selección de la empresa piloto fue por motivos de acceso y conveniencia, lo que facilitó la relación del entrevistado y el investigador para proceder con las mejoras del instrumento (Yin, 2003).

Todas las entrevistas fueron realizadas en una sesión independiente, con una duración aproximada de una hora; fueron grabadas y posteriormente transcritas. La información fue codificada, categorizada y analizada para poder extraer el contenido relevante de las entrevistas (Strauss y Corbin, 1998). Con las categorías determinadas se buscaron patrones y diferencias de comportamiento en los entrevistados, que fueron comparados con la literatura (Creswell, 2012).

La selección de las empresas entrevistadas se realizó a partir del informe publicado por la Revista Antioqueña de Economía y Desarrollo, de la Cámara de Comercio de Medellín para Antioquia, edición 15, donde se relacionaron las 500 empresas más grandes de Antioquia, y, bajo el criterio de muestreo no probabilístico purposive sampling, se seleccionaron los casos importantes de las grandes empresas privadas de Antioquia, que ayudan a responder las preguntas de investigación y que no serían de la misma calidad si se utilizaran otros medios (Greening, Barringer y Macy, 1996). Teniendo en cuenta que en Colombia, según la Ley 1450 de junio de 2011, las grandes empresas son las que cuentan con más de 200 empleados (Colombia, Congreso de la República, 2011), se eligieron empresas que cumplieran con dicha característica pero que a su vez contaran con desarrollos en riesgos estratégicos, dado que podían aportar información relevante y en gran cantidad para el estudio (Patton, 1990).

Los estudios cualitativos utilizan muestras pequeñas, pero que permiten estudiar el fenómeno en profundidad, dependiendo de sus contextos (Miles y Huberman, 1994); aunque no exista un número general de tamaño de la muestra para los estudios cualitativos (Marshall, Cardon, Poddar y Fontenot, 2013), se suele 
contar con tres y 10 participantes (Eisenhardt, 1989); para el presente estudio se cuenta con siete empresas, las cuales pertenecen a sectores diferentes, lo que facilita tener una mayor riqueza de la información recolectada. Para proteger la privacidad de las empresas, sus nombres fueron reemplazados por letras y con ello también se buscaba disminuir sesgos a la hora de las respuestas. En la Tabla 1 se presenta el perfil de las empresas que fueron entrevistadas:

TABLA 1

Perfil de los entrevistados

\begin{tabular}{llll}
\hline Empresa & Sector & Número de empleados & Años de fundada \\
\hline A & Bienes raíces & 1899 & 45 \\
B & Construcción & 10000 & 83 \\
C & Logístico & 4000 & 48 \\
D & Tecnología & 2700 & 82 \\
E & Financiero y tecnología & 290 & 16 \\
F & Energético & 653 & 22 \\
G & Agroindustrial & 7300 & 80 \\
\hline
\end{tabular}

Fuente: elaboración propia

\section{Resultados}

En el presente apartado se muestran los resultados obtenidos a partir de la información suministrada por los directivos entrevistados. En la Tabla 2 se puede observar la información de las estrategias de las empresas, basados en la teoría del reloj estratégico de Bowman (Johnson et al., 2006), con el fin de tener un mejor entendimiento de su relación con las medidas de tratamiento objeto de esta investigación.

TABLA 2

Estrategias adoptadas por las compañias entrevistadas

\begin{tabular}{lll}
\hline Empresa & Sector & Estrategia \\
\hline A & Bienes raíces & Diferenciación \\
B & Construcción & Diferenciación \\
C & Logístico & Diferenciación \\
D & Tecnología & Diferenciación segmentada \\
E & Financiero y tecnología & Diferenciación \\
F & Energético & Híbrida \\
G & Agroindustrial & Bajo precio \\
\hline
\end{tabular}

Fuente: elaboración propia

A partir de la información anterior y de acuerdo con las categorías de análisis encontradas en la literatura, como son el momento en que los riesgos estratégicos son identificados, su caracterización de acuerdo con el origen interno o externo y los tratamientos establecidos para enfrentar dichos riesgos, se relacionan extractos relevantes obtenidos en las entrevistas realizadas.

Con relación a la categoría de análisis correspondiente al momento en que el riesgo estratégico es identificado, pudo observarse que las empresas A, B, C, D, F y G reconocen el riesgo estratégico a partir de la ejecución estratégica. Así lo expresa el entrevistado de la empresa B, al afirmar que el "riesgo estratégico es todo aquello que pudiera impedir el logro de la estrategia”; por su parte, el entrevistado de la empresa E dijo que la 
identificación de sus riesgos estratégicos se da a partir de la formulación de la estrategia y el establecimiento de sus objetivos estratégicos. En sus palabras, "la estrategia está respondiendo a unos riesgos que nosotros identificamos [...] Planeación estratégica tenía los riesgos claros y con base en ellos estableció los objetivos estratégicos".

En cuanto a la categoría de análisis que obedece a la caracterización de origen de los riesgos estratégicos, los directivos entrevistados consideran importantes las variables internas y externas a la organización, y tienen en cuenta ambas clasificaciones para definir los riesgos estratégicos actuales. Sin embargo, estos coinciden en que el contexto externo es vital en el establecimiento y administración de los riesgos estratégicos, tal como lo manifiesta el entrevistado de la empresa $\mathrm{C}$ al afirmar que "el contexto externo es más importante que el mismo interno, porque se requiere mayor información de él en todo este proceso, saber qué pasa en el contexto macroeconómico, la industria y el mercado en los que nos movemos".

Igualmente, todos los entrevistados coinciden en la relevancia de conocer e interpretar las tendencias para la identificación y administración de los riesgos estratégicos, como señala el entrevistado de la empresa A, "es importante saber hacia dónde va el sector, el mercado, los consumidores. Pueden existir riesgos, que si no se miran como oportunidades, se presentan como dificultades a futuro".

Respecto a los riesgos estratégicos internos, los entrevistados están de acuerdo con que los riesgos críticos son aquellos relacionados con talento humano, definiciones inadecuadas en productos y proyectos que desencadenan la pérdida de clientes, y fallas relacionadas con la estructura y desarrollo organizacional. En este sentido, el entrevistado de la empresa D advierte que "realizar este análisis interno nos da muchas alertas y es necesario para nuestro análisis estratégico". Otros riesgos identificados son la concentración de clientes, de conocimiento, el financiero y el reputacional.

Una vez identificados y analizados los riesgos estratégicos, se procede a establecer medidas de tratamiento. Se encontró que los entrevistados conocen las medidas de control y de financiación para el tratamiento de los riesgos, pero por tratarse de riesgos concernientes al componente estratégico de sus organizaciones, los tratamientos utilizados están sujetos al análisis que se realice de cada riesgo en particular y en cada contexto.

$\mathrm{Al}$ respecto, la empresa $\mathrm{C}$ asegura que una vez analizados y evaluados sus riesgos estratégicos, "se decide si los asumimos, disminuimos frecuencia o impacto, retenemos o transferimos, y los asociamos a unos indicadores"; sin embargo, "estas medidas no son estáticas, sino que se asocian con planes de acción relacionados con la experiencia y la madurez de la compañía, y se incorporan iniciativas ya preexistentes que sirven para apoyar el logro de la estrategia”, y permiten que la organización pueda crear focos de atención, y a partir de la identificación y administración de riesgos estratégicos, "monitorear toda la realidad que se asocia a él", lo que representa un modelo de administración más efectivo para la organización. La empresa G, además, menciona la importancia de la prevención, sin embargo, hace énfasis en la necesidad de disminuir el impacto a través de medidas como "un plan de continuidad, la respuesta a la crisis o los procesos redundantes donde un tratamiento responda a varios riesgos".

Igualmente, los entrevistados están de acuerdo con afirmar que las medidas de tratamiento de los riesgos estratégicos parten de un claro conocimiento de la estrategia empresarial, así lo afirma la empresa A: "los tratamientos de los riesgos estratégicos tienen que nacer de la estrategia empresarial". Por eso, si bien en esta empresa la etapa de definir las medidas de tratamiento aún es incipiente, se han establecido algunos controles puntuales, por ejemplo, "para el riesgo de fallas en la estructura de negocio, se tiene como control que todo nuevo proyecto tenga una trazabilidad en sus procesos, documentación, comité de nuevos negocios y entrega de proyecto", y esto ha permitido crecer en forma organizada y agregar valor a los clientes y a los demás grupos de interés.

Así mismo, la empresa $\mathrm{G}$ afirma que, a partir de la apropiación de su estrategia, las respuestas a los riesgos que la afectan se crean desde iniciativas, proyectos y planes que contribuyan al cumplimiento de la estrategia. Y en esta vía, la empresa F sostiene que los riesgos estratégicos se gestionan a través de los procesos, por esto, 
a partir de la comprensión de su estrategia se implementan controles en proyectos y procesos, y esa sumatoria de tratamientos y controles "nos da como resultado la administración de los riesgos estratégicos".

La compañía E afirma que con base en el conocimiento de la estrategia y de sus riesgos se construye el mapa estratégico dividido en perspectivas con indicadores asociados. Agrega que "se hace seguimiento en forma trimestral en pro de que no se materialicen los riesgos de esas iniciativas estratégicas". La empresa D, que realiza esta misma práctica, implementa controles adicionales y "a partir de la arquitectura empresarial se revisa por qué los procesos no coinciden con la estrategia y, también, se realiza una auditoría estratégica que termina de cerrar la brecha entre lo que se formula y lo que se ejecuta". De este modo, ambas empresas avanzan en el cumplimiento de las estrategias anteriormente formuladas y contribuyen a su sostenibilidad y crecimiento.

Por otro lado, los directivos de las empresas B, C, D, E, F y G afirmaron que para obtener una ventaja competitiva, el tratamiento de los riesgos estratégicos también debe estar encaminado a la anticipación y al análisis de tendencias globales, por esto, el directivo de la empresa B menciona que planes de tratamiento como fit to grow, que se traduce como "optimizar la estructura de costos para crecer y penetrar nuevos mercados, con base en tendencias, representa una buena medida de tratamiento". En este sentido, estas compañías están dispuestas a tomar mayores riesgos y a realizar importantes inversiones en pro de conseguir grandes rendimientos. El directivo de la empresa D menciona que "cuando en las tendencias se identifican oportunidades ligadas con la estrategia, debe actuarse con mayor agresividad, una vez se obtiene la aprobación de la junta", y así evolucionar y perdurar en el tiempo, pues "la mirada no puede ser solo hacia aquello que inhibe la estrategia”. La empresa E menciona que la vigilancia tecnológica es un apoyo al revisar las tendencias y por ende "en el equipo de estrategia hay una persona dedicada a hacer vigilancia tecnológica para establecer la tipología de clientes, la penetración de canales digitales y las adquisiciones, entre otras, y eso soporta la apertura de nuevos mercados".

Así pues, los resultados obtenidos, si bien evidencian conocimiento de las medidas de tratamiento existentes para los riesgos estratégicos por parte de los directivos entrevistados, demuestran que su implementación depende de las decisiones estratégicas que se toman de acuerdo con cada contexto empresarial y con el momento en el que se encuentra cada organización. También se percibe que las empresas entrevistadas cuentan con un proceso de planeación estratégica que facilita la identificación y posterior administración de los riesgos estratégicos. Además, puede notarse una actitud menos conservadora al momento de tomar decisiones frente al tratamiento de estos riesgos, pues si bien se busca proteger el valor de sus organizaciones, también se observa la intención de implementar nuevas medidas que permitan crearlo.

Igualmente, puede verse que, aunque el proceso de implementación de las medidas de tratamiento en algunas de las empresas entrevistadas ha sido más trabajado, en todas existe la conciencia acerca de la importancia de consolidar, en mayor medida, esta etapa dentro del sistema de administración de riesgos estratégicos.

\section{Discusión y conclusiones}

En la presente investigación se analizaron, desde la teoría, las medidas de tratamiento existentes para administrar los riesgos estratégicos: prevenir, proteger, evitar, aceptar, retener, transferir y perseguir, pues a partir de ellas se logra contrarrestar su efecto negativo (ISO 31000, 2018; COSO, 2016; Slywotzky y Drizk, 2005; AS NZS 4360, 2004) o bien capitalizar los beneficios derivados de su materialización (Jnr y Pa, 2016).

A la luz de lo encontrado en la literatura se analizaron los aportes de los directivos entrevistados y se evidenció su conciencia ante el riesgo estratégico, al proceso que comprende su administración y a las medidas de tratamiento existentes, aunque resaltan que, en lo concerniente al ámbito estratégico, su aplicación es diferente de otro tipo de riesgos. Estos resultados apoyan estudios que indican que el tratamiento de los riesgos 
estratégicos debe responder al carácter dinámico de la estrategia y a las características únicas de la organización (Frigo y Anderson, 2011).

En la presente investigación se identificó, desde la teoría, que existen características propias de la empresa, como la estrategia, la cultura y el modelo de negocio. En este estudio se profundizó en la relación de la estrategia con las medidas de tratamiento de sus riesgos. Por tanto, la pertenencia de las empresas entrevistadas a diferentes sectores económicos, sumada a la estrategia elegida para competir en el mercado, hace que surjan diferentes riesgos estratégicos, y las medidas para enfrentarlos varían en pro de lograr esa posición realizable y sostenible que, a su vez, garantice la consecución de los objetivos estratégicos (McConnell, 2016; COSO, 2016).

Como futuras líneas de investigación se propone analizar si las conductas de las personas, sus valores y creencias, como componentes de la cultura organizacional (Pojasek, 2017), podrían convertirse en base para diseñar tratamientos a los riesgos estratégicos y contribuir con el cumplimiento de los objetivos. Adicionalmente, estudiar cómo los componentes del modelo de negocio, que determinan los aspectos clave de la empresa para poder ofrecer la propuesta de valor (Osterwalder y Pigneur, 2010), se podrían utilizar en futuras investigaciones para conocer la relación con los tratamientos internos que implementan las empresas para responder a sus riesgos estratégicos.

Del mismo modo, en las siete empresas entrevistadas se encontró que, para definir las medidas de tratamiento de los riesgos estratégicos, el primer paso debe ser el conocimiento y la apropiación de la estrategia, pues a partir de allí se pueden alinear planes que efectivamente aporten a la consecución de los objetivos planteados. Esto responde parcialmente al planteamiento teórico que indica que, la adecuada administración del riesgo estratégico implica el conocimiento tanto de la estrategia como de los riesgos asociados con su formulación y ejecución (Mohammed y Sykes, 2013), debido a que solo una de las empresas entrevistadas reconoce los riesgos y determina medidas de tratamiento a partir de la formulación estratégica; las seis restantes, lo hacen a partir de la ejecución. En este punto se resalta que las respuestas suministradas sobre la responsabilidad de la alta dirección de definir tratamientos para sus riesgos estratégicos, son acordes con los hallazgos en la literatura, que afirma que la administración de estos riesgos es una habilidad necesaria de la alta dirección (Frigo y Anderson, 2012).

Además, al establecer medidas de tratamiento para los riesgos estratégicos se busca incrementar las oportunidades y reducir las potenciales amenazas (Toulabi y Toulabi, 2013), para aportar al crecimiento de las organizaciones, se encontró que la implementación de tales medidas en las empresas estudiadas ha propiciado un modelo de administración más adecuado, organizado y acorde con sus planes, políticas y necesidades estratégicas. Igualmente, ha permitido la protección y creación de valor para los diferentes grupos de interés, y también la sostenibilidad, el crecimiento y la permanente evolución. Lo anterior obedece al planteamiento que señala la importancia de la administración de este tipo de riesgos en el éxito empresarial, de lo contrario, puede conducir al fracaso de la estrategia y a la quiebra (McConnell, 2016).

Por otro lado, se observó que las empresas entrevistadas consideran importante la asignación de recursos y el desarrollo de capacidades para el tratamiento de los riesgos aludidos, por lo que se considera conveniente utilizar como referente la teoría de recursos y capacidades (Barney y Arikan, 2001) para emprender investigaciones futuras que permitan comprender cómo dichos elementos contribuyen a la adecuada administración de estos riesgos.

Además, se encontró que en seis de las empresas entrevistadas existe una concepción menos conservadora del riesgo, por lo cual implementan disposiciones tendientes a perseguirlo y a lograr transformaciones en busca de oportunidades. Dentro de estas empresas, dos pertenecen al sector de las tecnologías, por lo cual se sugiere plantear investigaciones futuras para conocer si esta mirada del riesgo como oportunidad se debe a una característica propia de este sector; además de otras investigaciones que profundicen en el conocimiento del riesgo positivo (Slywotzky, 2008). 


\section{Referencias bibliográficas}

AIRMIC, Alarm y IRM (2010). A Structured Approach to Enterprise Risk Management (ERM) and the Requirements of ISO 31000 .

Andersen, T. J., Garvey, M. y Roggi, O. (2014). Managing Risk and Opportunity. The Governance of Strategic RiskTaking. Oxford.

Andersen, T. J. y Schroder, P. W. (2010). Strategic Risk Management Practice: How to Deal with Major Corporate Exposures. Cambridge, United Kingdom: Cambridge University Press.

Andrews, K. (1984). El concepto de estrategia de la empresa. Madrid: Ediciones Orbis.

AS NZS (2004). Australian/New Zealand Standard, Risk Management.

Barney, J. y Arikan, A. (2001). Resource-Based View: Origins and Implications. En M. Hitt, R. Freeman y J. Harrison (eds). The Blackwell Handbook of Strategic Management (pp. 124-188). Malden, Massachusetts: Blackwell.

Beck, U. (2002). La sociedad del riesgo global. Madrid: Siglo Veintiuno.

Bravo, O. y Sánchez, M. (2009). Gestión Integral de Riesgos. Tomo 1. Bogotá: Bravo y Sánchez.

Bromiley, P., Rau, D. y McShane, M. (2016). Can Strategic Risk Management Contribute to Enterprise Risk Management? A Strategic Management Perspective. In T. J. Andersen (Ed.), The Routledge Companion to Strategic Risk Management (pp. 140-156).

Byrne, P. y Durant-Law, G. (2007). A Strategic Risk Based Approach to Regulating Technologies and Vulnerabilities. In Technology and Society, 2007. ISTAS 2007. IEEE International Symposium on (pp. 1-11). IEEE.

Calandro, J. (2015). A Leader’s Guide to Strategic Risk Management. Strategy \& Leadership, 43 (1), 26-35.

Colombia, Congreso de la República (2011). Ley 1450, por la cual se expide el Plan Nacional de Desarrollo, 2010-2014. Diario oficial 48102, del 16 de junio. Recuperado de http://www.alcaldiabogota.gov.co/sisjur/normas/Norma 1.jsp?i=43101

Committee of Sponsoring Organizations of the Treadway Commission (COSO). (2017). Enterprise Risk Management Aligning Risk with Strategy and Performance. Executive Summary, 3.

Creswell, J. W. (2012). Qualitative inquiry and research design: Choosing among five approaches. California: Sage Publications.

Deloitte. (2016). Riesgo Estratégico. La piedra angularpara la transformación del riesgo. Recuperado de http://deloitt e.wsj.com/riskandcompliance/files/2013/10/exploring_strategic_risk.pdf

Edwards, J. S. y Taborda, E. R. (2016). Using Knowledge Management to Give Context to Analytics and Big Data and Reduce Strategic Risk. Procedia Computer Science, 99, 36-49.

Eisenhardt, K. (1989). Building Theories from Case Study Research. Academy of Management Review, 14 (4), 532-550.

Erlandson, D., Harris, E., Skipper, B. y Allen, D. S. (1993). Doing Naturalistic Inquiry, Londres: Sage.

Frigo, M. y Anderson, R. J. (2012).Strategic Risk Management: The New Core Competency. John Wiley \& Sons Limited.

Frigo, M. L. y Anderson, R. J. (2011). Strategic Risk Management: A Foundation for Improving Enterprise Risk Management and Governance. Journal of Corporate Accounting \& Finance, 22 (3), 81-88.

Frigo, M. L. y Anderson, R. J. (2009). Strategic Risk Assessment. Strategic finance, 25-33.

Greening, D. W., Barringer, B. R. y Macy, G. (1996). A Qualitative Study of Managerial Challenges Facing Small Business Geographic Expansion. Journal of Business Venturing, 11 (4), 233-256.

Hardy, C. y Maguire, S. (2016). Organizing Risk: Discourse, Power, and "Riskification". Academy of Management Review, 41 (1), 80-108.

Hernández, R., Fernández, C. y Baptista, P. (2010). Metodología de la investigación (5th ed.). México D.F.: McGrawHill.

International Organization for Standardization (ISO) (2018). ISO 31000. Risk Management-Guidelines. Suiza: ISO.

Jnr, B. A. y Pa, N. C. (2016). Mitigating Operational, Technical and Strategic Risk in it Through Knowledge Codification Technique. Journal Teknologi, 78 (8-2), 179-192. 
Johnson, G., Scholes, K., Whittington, R., López, Y. M. y Mazagatos, V. B. (2006). Dirección estratégica (Vol. 5). Prentice Hall.

Kaplan, R. S. y Norton, D. P. (2008). The Execution Premium: Linking Strategy to Operations for Competitive Advantage. Massachusetts: Harvard Business Press.

Knight, F. H. (1921). Risk, Uncertainty and Profit. New York: Hart, Schaffner and Marx.

Louisot, J. P. y Ketcham, C. (2014). Enterprise Risk Management: Issues and Cases. West Sussex, United Kingdom: John Wiley and Sons Ltd.

Marshall, B., Cardon, P., Poddar, A. y Fontenot, R. (2013). Does Sample Size Matter in Qualitative Research?: a Review of Qualitative Interviews in IS Research. Journal of Computer Information Systems, 54 (1), 11-22.

McConnell. (2016). Strategic Risk Management. London, England: Risk Books.

Mejía, R. (2013). Identificación de riesgos. Medellín: Universidad Eafit.

Mejía, R. (2006). Administración de riesgos: Un enfoque empresarial. Medellín: Universidad Eafit.

Miles, M. B. y Huberman, A. M. (1994). Qualitative Data Analysis: An Expanded Sourcebook. Thousand Oaks, CA: Sage Publications.

Mohammed, A. y Sykes, R. (2013). Afilado de Gestión Estratégica del Riesgo. Pricewaterhouse Coopers. Recuperado de http://www.pwc.com/gx/en/governance-risk-compliance-consulting-services/resilience/publications/pdfs/ issue 1/sharpening_strategic_risk_management.pdf

Osterwalder, A., y Pigneur, Y. (2010). Business model generation: a handbook for visionaries, game changers, and challengers. New Yersey: EE.UU.: John Wiley \& Sons.

Patton, M. Q. (1990). Qualitative Evaluation and Research Methods. Londres: Sage.

Pojasek, R. B. (2017). Organizational Risk Management and Sustainability: A practical step-by-step guide. Florida, EE.UU.: CRC Press.

Porter, M. (1991). La ventaja competitiva de las naciones. Buenos Aires: Vergara.

Rubin, H. y Rubin, I. (1995). Qualitative Interviewing: The Art of Hearing Data. Thousand Oaks, California: Sage.

Slywotzky, A. J. (2008). Riesgo positivo. Bogotá: Norma.

Slywotzky, A. y Drzik, J. (2005). Contrarrestar el mayor de todos los riesgos. Harvard Business Review, 83 (4), 58-69.

Slywotzky, A. (2004). Exploring the Strategic Risk Frontier. Strategy \& Leadership, 32 (6), 11-19.

Song, H. y Yang, S. (2015). Study on Strategic Risk Evaluation Model of Power Grid Enterprises Based on Fuzzy Evaluation Method. In Logistics, Informatics and Service Sciences (LISS), 2015 International Conference on (1-4). IEEE.

Strauss, A. L. y Corbin, J. (1998). Basics of Qualitative Research: Techniques and Procedures for Developing Grounded Theory. Book (Vol. 2nd). Thousand Oaks, California: Sage.

Toulabi, M. y Toulabi, A. (2013). Strategic Risk Management with the Approach Huge in BOT Projects. Advances in Environmental Biology. Volume 7, Issue 8.

Vargas Jiménez, I. (2012). La entrevista en la investigación cualitativa: nuevas tendencias y retos. Calidad en la Educación Superior, 3 (1), 119-139.

Yin, R. K. (2003). Case Study Research: Concepts and Methods. Thousand Oaks, California: Sage.

\section{Licencia Creative Commons CC BY 4.0}

Para citar este artículo: Jaraba, I., Nuñez, M. A. y Villanueva, E. (2017). Riesgos estratégicos. Un estudio de las medidas de tratamiento implementadas por las grandes empresas privadas de Antioquia, Colombia. Cuadernos de Contabilidad, 19(47), 171-181. https://doi.org/10.11144/Javeriana.cc19-47.reem 